\title{
NONLINEAR MODEL REFERENCE ADAPTIVE CONTROL
}

\author{
J. M. SKOWRONSKI ${ }^{1}$
}

(Received 11 September 1985; revised 21 October 1985)

\begin{abstract}
The known linear model reference adaptive control (MRAC) technique is extended to cover nonlinear and nonlinearizable systems (several equilibria, etc) and used to stabilize the system about a model. The method proposed applies the same Liapunov Design Technique but avoids the classical error equation. Instead it operates in the product of the state spaces of plant and model, aiming at convergence to a diagonal set. Control program, Liapunov functions and adaptive law are specified. The case is illustrated on a two-degrees of freedom robotic manipulator.
\end{abstract}

\section{Introduction}

The range of applications of the model reference adaptive control (MRAC) widens steadily: from autopilots in the sixties, cf. [9], particularly VTOL, up to recent uses in biology, cf. [12], and robotics, cf. [3], [1]. Consequently the literature on MRAC is vast, see for review [6]. The broadest avenue of research bases on the so-called Liapunov Design Technique or Liapunov Synthesis methods, cf. [5], [13]. Unfortunately, in spite of the fact that the real world is nonlinear, and in spite of the known nonlinear capabilities of the Liapunov theory, the existing results refer, with very few exceptions, to linear or linearizable systems. The technique used is that of subtracting the state equations of the plant from those of the model, thus producing the so-called error equation. Then the asymptotic stability of its trivial solution (zero-error) secures the convergence of both states and parameters of the plant and the model. For nonlinearizable systems, such a subtraction is not possible in explicit terms. Thus we propose to abandon the error equation, and instead to achieve the required convergence by

\footnotetext{
${ }^{1}$ Department of Mathematics, University of Queensland, St. Lucia, Qld, 4067, Australia.

(c) Copyright Australian Mathematical Society 1986, Serial-fee code 0334-2700/86
} 
securing the asymptotic stability, or / and its finite-time counterpart, of a diagonal set in the Cartesian product of the states spaces of plant and model. This produces the same effect, but allows arbitrary nonlinearity and noncompatible state-space patterns (several different equilibria, etc) between plant and model. The method comes close to that proposed in [10], and adopts a similar technique to that used for identification in [11], [12].

Moreover, the present MRAC theory means model-tracking only, while in reality one may as often need model-avoidance, cf. [7], [8], as well as many other variations of the theme, e.g. tracking the avoidance in prespecified time intervals, tracking one model and avoiding another ..., etc. With the method discussed here one should be able to achieve satisfactory results in all such studies. Obviously for avoidance we have to show divergence rather than convergence to the diagonal.

\section{Linear tracking}

It seems useful to begin with a brief outline of the linear technique. Let the plant be described by the state equations

$$
\dot{x}=A(t) x+B(t) u,
$$

where $x(t)=\left(x_{1}(t), \ldots, x_{N}(t)\right)^{T} \in \mathbf{R}^{N}$ is the state vector, $t \in\left[t_{0}, \infty\right), t_{0}$ is the initial instant, and $A, B$ are the system and input matrices $N \times N, N \times r$, respectively, while $u(t)=\left(u_{1}(t), \ldots, u_{r}(t)\right)^{T} \in U \subset \mathbf{R}^{r}$ is the control vector admissible if selected from within the compact set $U$ of constraints on control values. The selection of $u(t)$ is due to a feedback control program which aims to stabilize the plant during the tracking. The reference model to be followed is given as

$$
\dot{x}_{m}=A_{m} x_{m}+B_{m} u_{m}
$$

where $x_{m}(t) \in \mathbf{R}^{N}, t \geqslant t_{0}$, while $A_{m}, B_{m}$ are constant $N \times N, N \times r$ matrices and $u_{m}(t) \in U \subset \mathbf{R}^{r}$ has been selected to make the model attain some objective behaviour.

We denote the state error by $e(t)=x_{m}(t)-x(t), t \geqslant t_{0}$, and the parameter differences by $A_{e}(t)=A_{m}-A(t), B_{e}(t)=B_{m}-B(t)$, where $A(t), B(t)$ are adjustable. Subtracting (2.1) from (2.2) produces the error equation

$$
\dot{e}=A_{m} e+A_{e}(t) x+B_{e}(t) u .
$$


The plant follows the model, or more exactly, the plant converges asymptotically to the model, if

$$
e(t) \rightarrow 0, A_{e}(t) \rightarrow 0, B_{e}(t) \rightarrow 0 \text { as } t \rightarrow \infty,
$$

which thus becomes the tracking objective. As is well known, it may be achieved via some Liapunov function $V\left(e, A_{e}, B_{e}\right)$ which is positive definite about the single equilibrium of (2.3) and possesses a negative-definite derivative along the trajectories of (2.3), while also having a nesting property about the equilibrium. The obvious candidate is the square form

$$
V\left(e, A_{e}, B_{e}\right)=e^{T} P e+\sum_{i=1}^{N} \sum_{j=1}^{N} a_{e l j}^{2}+\sum_{i=1}^{N} \sum_{k=1}^{r} b_{e i k}^{2},
$$

where $P=\left(p_{1 j}\right)$ is a positive-definite symmetric matrix and $a_{e t j}, b_{e l k}$ are components of $A_{e}(t), B_{e}(t)$, respectively. The only property to check is the negative derivative, the other two being obviously satisfied. Differentiating (2.5) and substituting (2.3), we have

$$
\begin{aligned}
\dot{V}(t)= & \left(A_{m} e+A_{e} x+b_{e} u\right)^{T} P e+e^{T} P\left(A_{m} e+A_{e} x+B_{e} u\right) \\
& +2 \sum_{i} \sum_{j} a_{e i j} \cdot \dot{a}_{e t j}+2 \sum_{t} \sum_{k} b_{e i k} \cdot \dot{b}_{e i k},
\end{aligned}
$$

or

$$
\begin{aligned}
\dot{V}(t)= & e^{T}\left(A_{m}^{T} P+P A_{m}\right) e+x^{T} A_{e}^{T}\left(P+P^{T}\right) e \\
& +u^{T} B_{e}^{T}\left(P+P^{T}\right) e+2 \sum_{i} \sum_{J} a_{e \imath \jmath} \cdot \dot{a}_{e i j}+2 \sum_{l} \sum_{k} b_{e t k} \cdot \dot{b}_{e t k} .
\end{aligned}
$$

The negative definiteness of the first term on the right-hand side of (2.6) follows immediately from the so-called Liapunov Matrix Equation:

$$
A_{m}^{T} P+P A_{m}=-Q,
$$

where $Q$ is a positive-definite matrix, which however need not be determined, if a stable reference model was chosen. Granted the above, it suffices to reduce the other terms of (2.6) to zero. Simple calculation shows that the latter happens if the adaptive laws

$$
\left.\begin{array}{l}
\dot{a}_{e i j}=-\frac{1}{2} x_{j} \sum_{\sigma=1}^{N}\left(p_{i \sigma}+p_{\sigma i}\right) e_{\sigma}, \\
\dot{b}_{e i x}=-\frac{1}{2} u_{k} \sum_{\sigma=1}^{r}\left(p_{i \sigma}+p_{\sigma i}\right) e_{\sigma},
\end{array}\right\}
$$


hold. Note that since $A_{m}, B_{m}$ are constant, we have $\dot{a}_{e i j}=\dot{a}_{i j}, \dot{b}_{e l x}=\dot{b}_{t k}$. It is then obvious that suitable solutions to $(2.8)$ provide the negative-definite $\dot{V}(t)$, which implies the required asymptotic stability (2.4).

\section{Nonlinear tracking}

We let now the plant take the general form

$$
\dot{x}=f(x, u ; \lambda),
$$

where $f=\left(f_{1}, \ldots, f_{N}\right)^{T}$ is the $N$-vector of functions $f_{i}(x, u, \lambda), i=1, \ldots, N$, suitable for securing the unique solutions through each point $x^{0}=x(0)$ in a given bounded subset $\Delta$ of $\mathbf{R}^{N}$, provided suitable values of $u, \lambda, w$ are given. The $l$-vector parameter $\lambda(t)=\left(\lambda_{1}(t), \ldots, \lambda_{l}(t)\right)^{T} \in \Lambda \subset \mathbf{R}^{\prime}$ represents the adjustable parameters of the plant, with their values bounded in a compact set $\Lambda \subset \mathbf{R}^{\prime}$. We let $l \leqslant N$. The control vector $u(t)=\left(u_{1}(t), \ldots, u_{r}(t)\right)^{T} \in U \subset \mathbf{R}^{r}$ is to be chosen by a feedback and signal adaptable control program $u(t)=P(x(t), \lambda(t)), t \geqslant t_{0}$, in order to attain a specified objective for (3.1) apart from the tracking. To focus attention on the tracking we choose a simple objective of stabilization: Lagrange stability in some region enclosing all the equilibria. The control program is admissible if it produces $u(t)$ that allows the solutions to (3.1) on $\Delta$.

Considering our aims, it does not narrow generality to assume that (3.1) has a finite number of isolated equilibria $x(t) \equiv x^{e}=$ constant, all contained in $\Delta$.

The reference model is taken again as a nonlinear system

$$
\dot{x}_{m}=f_{m}\left(x_{m}, u_{m} ; \lambda_{m}\right),
$$

with $x_{m}(t) \in \Delta \subset \mathbf{R}^{N}, u_{m}(t) \in U \subset \mathbf{R}^{r}$ selected by a control program that makes (3.2) perform a specific task in the state space, possibly done within the scope of the Liapunov theory, i.e. with a specific Liapunov function. To focus attention on something definite, let us make $u_{m}$ such that (3.2) is asymptotically stable about the origin, with given Liapunov function $V_{m}\left(x_{m}\right)$ which is positive definite, nesting about the origin, and has negative definite derivative $\dot{V}_{m}\left(x_{m}(t)\right)$ along solutions of (3.2).

Let us now introduce the $2 N$-vector $X(t) \stackrel{\Delta}{=}\left(x(t), x_{m}(t)\right)^{T}$ in the set $\Delta^{2} \stackrel{\Delta}{=} \Delta \times \Delta$ and denote $\alpha(t)=\lambda(t)-\lambda_{m}, t \geqslant t_{0}$ ranging in $\Lambda \subset \mathbf{R}^{l}$. Hence also $(X(t), \alpha(t))^{T}$ ranges in $\Delta^{2} \times \Lambda \subset \mathbf{R}^{2 N+1}$. Then we define the "diagonal"

$$
M=\left\{(X, \alpha)^{T} \in \Delta^{2} \times \Lambda \mid x=x_{m}, \alpha=0\right\}
$$


and its $\eta(>0)$-neighbourhood by

$$
M_{\eta}=\left\{(X, \alpha)^{T} \in \Delta^{2} \times \Lambda\left|\left\|x-x_{m}\right\| \leqslant \eta,\right| \alpha \mid \leqslant \eta\right\}
$$

where $\|\cdot\|$ is any norm in $\mathbf{R}^{2 N+!}$.

DeFinition 3.1. We shall say that the plant (3.1) is stabilized in $\Delta$ and tracks the model (3.2) with $\eta>0$ accuracy, if there is an admissible control program $u(t)=P(x(t), \lambda(t)), t \geqslant t_{0}$ such that

(A) $\left(X^{0}, \alpha^{0}\right)^{T} \in \Delta^{2} \times \Lambda \Rightarrow(X(t), \alpha(t))^{T} \in \Delta^{2} \times \Lambda, \forall t \geqslant t_{0}$;

(B) there are constants $\eta>0, T>0$ such that

$$
\left(X^{0}, \alpha^{0}\right)^{T} \in \Delta^{2} \times \Lambda \Rightarrow(X(t), \alpha(t))^{T} \in M_{\eta}, \forall t \geqslant T .
$$

\section{Sufficient conditions}

Let $\mathscr{N}\left[\partial\left(\Delta^{2} \times \Lambda\right)\right]$ be a neighbourhood of the boundary $\partial\left(\Delta^{2} \times \Lambda\right)$ of the region $\Delta^{2} \times \Lambda$ and define $N_{\varepsilon} \stackrel{\Delta}{=} \mathcal{N}\left[\partial\left(\Delta^{2} \times \Lambda\right)\right] \cap \overline{\Delta^{2} \times \Lambda}, C M_{\eta}=\left(\Delta^{2} \times \Lambda\right)-$ $M_{\eta}, S$ (open) $\supset \overline{C M_{\eta}}$ and such that $S \cap M=\varnothing$. Furthermore introduce two functions $V_{S}: N_{\varepsilon} \rightarrow \mathbf{R}, V_{\eta}: S \rightarrow \mathbf{R}$ with

$$
\begin{aligned}
& v_{s} \stackrel{\Delta}{=} \inf \left\{V_{S}(X, \alpha) \mid(X, \alpha) \in \partial\left(\Delta^{2} \times \Lambda\right)\right\}, \\
& v_{\eta}^{-} \stackrel{\Delta}{=} \inf \left\{V_{\eta}(X, \alpha) \mid(X, \alpha) \in \partial M_{\eta} \cap \overline{C M_{\eta}}\right\}, \\
& v_{\eta}^{+} \stackrel{\Delta}{=} \sup \left\{V_{\eta}(X, \alpha) \mid(X, \alpha) \in \partial\left(\Delta^{2} \times \Lambda\right) \cap \overline{C M_{\eta}}\right\} .
\end{aligned}
$$

THEOREM 4.1. Definition 3.1 holds if, given $\Delta, \Lambda, \eta$ there is an admissible $P(x, \lambda)$ and a pair of functions $V_{S}, V_{\eta}$ such that for all $X, \alpha$ in their domains we have

(i) $V_{S}(X, \alpha) \leqslant V_{S}, \forall(X, \alpha) \in N_{e}$;

(ii) $\nabla V_{S}(X, \alpha) \cdot\left[f(x, u, \lambda), f_{m}\left(x_{m}, u_{m}, \lambda_{m}\right), \dot{\alpha}\right]^{T}<0$;

(iii) $0 \leqslant V_{\eta}(X, \alpha) \leqslant v_{\eta}^{+}, v_{\eta}^{+}>0, \forall(X, \alpha) \in \overline{C M_{\eta}}$;

(iv) $V_{\eta}(X, \alpha) \leqslant v_{\eta}^{-}, \forall(X, \alpha) \in S \cap M_{\eta}$;

(v) there is a constant $c>0$ such that for all $(X, \alpha) \in \overline{C M_{\eta}}$, $\nabla V_{\eta}(X, \alpha) \cdot\left[f(x, u, \lambda), f_{m}\left(x_{m}, \lambda_{m}, u_{m}\right), \dot{\alpha}\right]^{T} \leqslant-c$.

Proof. No motion $(X(t), \alpha(t))^{T}=\left(x(t), x_{m}(t), \alpha(t)\right)^{T}$, where $x(t), x_{m}(t)$ are solutions of (3.1), (3.2) respectively, leaves $\Delta^{2} \times \Lambda$ unless passing through $N_{\varepsilon}$, so we consider $\left(X^{0}, \alpha^{0}\right) \in \mathscr{N}_{\varepsilon}$ and suppose the corresponding motion crosses $\partial\left(\Delta^{2}\right.$ $\times \Lambda)$. Then there is $t_{1} \geqslant 0$ such that $\left(X\left(t_{1}\right), \alpha\left(t_{1}\right)\right)^{T} \in \partial\left(\Delta^{2} \times \Lambda\right)$ and by (i), 
$V_{S}\left(X\left(t_{1}\right), \alpha\left(t_{1}\right)\right) \geqslant v_{S} \geqslant V_{S}\left(X^{0}, \alpha^{0}\right)$ contradicting (ii); hence no such motion leaves $\Delta^{2} \times \Lambda$ and the requirement (A) of Definition 3.1 is satisfied.

Next we show that the motions from $C M_{\eta}$ may not stay there indefinitely. Indeed, consider arbitrary such motion. By $(v), \dot{V}_{\eta}(X(t), \alpha(t)) \leqslant-c$. Integrating this over $[0, t] \subset \mathbf{R}$, we obtain the estimate

$$
t \leqslant \frac{1}{c}\left[V_{\eta}\left(X^{0}, \alpha^{0}\right)-V_{\eta}(X, \alpha)\right]
$$

Note that by (iii), $V_{\eta}(X, \alpha) \geqslant 0$ and $V_{\eta}\left(X^{0}, \alpha^{0}\right)-v_{\eta}^{+} \leqslant 0$ whence $V_{\eta}\left(X^{0}, \alpha^{0}\right)-$ $V_{\eta}(X, \alpha) \leqslant v_{\eta}^{+}$, allowing us to rewrite (4.1) as $t \leqslant v_{\eta}^{+} / c$. Hence there is $T=v_{\eta}^{+} / c$, depending upon the diameter of $C M_{\eta}$ and nothing else, such that for $t \geqslant T$ the motion leaves $C M_{\eta}$. As it must not leave $\Delta^{2} \times \Lambda$, then it must enter $M_{\eta}$ and be there at some $t_{\eta}=T+\tau, \tau>0$. There is no return to $\overline{C M_{\eta}}$. Indeed, if there were $t_{2}>t_{\eta}$ such that $\left(X\left(t_{2}\right), \alpha\left(t_{2}\right)\right)^{T} \in \partial M_{\eta}$, then by (iv) we would have $V_{\eta}\left(X\left(t_{2}\right), \alpha\left(t_{2}\right)\right) \geqslant v_{\eta}^{-} \geqslant V_{\eta}\left(X\left(t_{\eta}\right), \alpha\left(t_{\eta}\right)\right)$, contradicting (v). The above secures (B) of Definition 3.1, thus completing the proof.

Obviously $T$ may be calculated from $T=v_{\eta}^{+} / c$ if $V_{\eta}(\cdot)$ and $c$ are given. Suppose now that an a priori value of $t$ was demanded. We have

Corollary 4.1. Definition 3.1 holds effective after a given $T$, if Theorem 4.1 is satisfied with the condition (v) replaced by

$$
\nabla V_{\eta}(X, \alpha) \cdot\left[f(x, u, \lambda), f_{m}\left(x_{m}, u_{m}, \lambda_{m}\right)\right] \leqslant-v_{\eta}^{+} / T
$$

The proof follows by choosing $c=v_{\eta}^{+} / T$.

\section{Liapunov functions and adaptive laws}

We have assumed the Liapunov function $V_{m}\left(x_{m}\right)$ for the asumptotic stability of the model to be known. The standard asymptotic stability conditions require it to be positive definite, monotone increasing and nesting about $V(0)=0$, while the derivative satisfies

$$
\dot{V}_{m}\left(x_{m}(t)\right) \leqslant-c \text {. }
$$

We let $V_{m}$ have these properties. Let us now set up $l=N$, and propose

$$
V_{S}(X, \alpha)=V_{m}(x)+V_{m}\left(x_{m}\right)+a \cdot \alpha
$$

and

$$
V_{\eta}(X, \alpha)=\left\{\begin{array}{ll}
\left|V_{m}(x)-V_{m}\left(x_{m}\right)\right|+a \cdot \alpha, & (X, \alpha) \in \overline{C M_{\eta}} \\
0, & (X, \alpha) \in M_{\eta}
\end{array}\right\}
$$


where $a=\left(\operatorname{sign} \alpha_{1}, \ldots, \operatorname{sign} \alpha_{N}\right)$. In view of our assumptions on $V_{m}$, the conditions (i), (iii), (iv) hold in an obvious way, and all we have to show is (ii) and (v).

Consider the derivative of (5.2)

$$
\dot{V}_{S}(X(t), \alpha(t))=\dot{V}_{m}(x(t))+\dot{V}_{m}\left(x_{m}(t)\right)+a \cdot \dot{\alpha},
$$

and form the adaptive law

$$
\dot{\alpha}_{i}=\frac{1}{\operatorname{sign} \alpha_{i}} \frac{\partial V_{m}\left(x_{m}\right)}{\partial x_{m i}} \cdot f_{m i}\left(x_{m}, u_{m}, \lambda_{m}\right), \quad i=1, \ldots, N,
$$

which, in view of (5.1) implies

$$
a \cdot \dot{\alpha}=\dot{V}_{m}\left(x_{m}(t)\right) \leqslant-c,
$$

in agreement with our required convergence of $\alpha(t)$. Then let us design the control program $P(x, \lambda)$ from the following condition

$$
\nabla V_{m}(x) \cdot f(x, P(x, \lambda), \lambda)=-c .
$$

Note that (5.7) gives as specific a condition as we can get without specifying $V_{m}(\cdot), f(\cdot)$ any closer. The latter may be done in case studies, and then $P(x, \lambda)$ may be determined in an explicit form, calculated from (5.7).

Then, from (5.4) by (5.1), (5.6) and (5.7) we have $\dot{V}_{S}(X(t),(t))<0$, thus satisfying (ii). Now we have to show (v). Let us introduce the sets in $\Delta^{2}-M_{\eta}: \mathscr{A}$ : $V_{m}(x)-V_{m}\left(x_{m}\right)>0, \mathscr{B}: V_{m}(x)-V_{m}\left(x_{m}\right)<0$, and differentiate (5.3)

$$
\dot{V}_{\eta}(X(t), \alpha(t))= \begin{cases}\dot{V}_{m}(x)-\dot{V}_{m}\left(x_{m}\right)+a \cdot \dot{\alpha}, & X \in \mathscr{A} ; \\ \dot{V}_{m}\left(x_{m}\right)-\dot{V}_{m}(x)+a \cdot \dot{\alpha}, & X \in \mathscr{B} .\end{cases}
$$

On substituting (5.6), (5.1) and (5.7) we conclude

$$
\dot{V}_{\eta}(X(t), \alpha(t))= \begin{cases}\dot{V}_{m}(x)=-c, & X \in \mathscr{A} \\ 2 \dot{V}_{m}\left(x_{m}\right)-\dot{V}_{m}(x) \leqslant-c, & X \in \mathscr{B}\end{cases}
$$

which satisfies condition (v), thus concluding our investigation.

\section{Application to robotic manipulator}

A $n$-joint manipulator is representable in terms of a combination of modular two degrees of freedom units, as displayed in Figure 1. We take the Lagrange (or joint) coordinates $q_{1}=\theta, q_{2}=r$ as indicated, with $r_{1}=$ const. at the rotary joint 1. Elementary mechanics gives the kinetic energy as

$$
T=\frac{1}{2} m_{1} r_{1}^{2} \dot{q}_{1}^{2}+\frac{1}{2} m^{2}\left(\dot{q}_{2}^{2}+\dot{q}_{2} \dot{q}_{1}^{2}\right)
$$




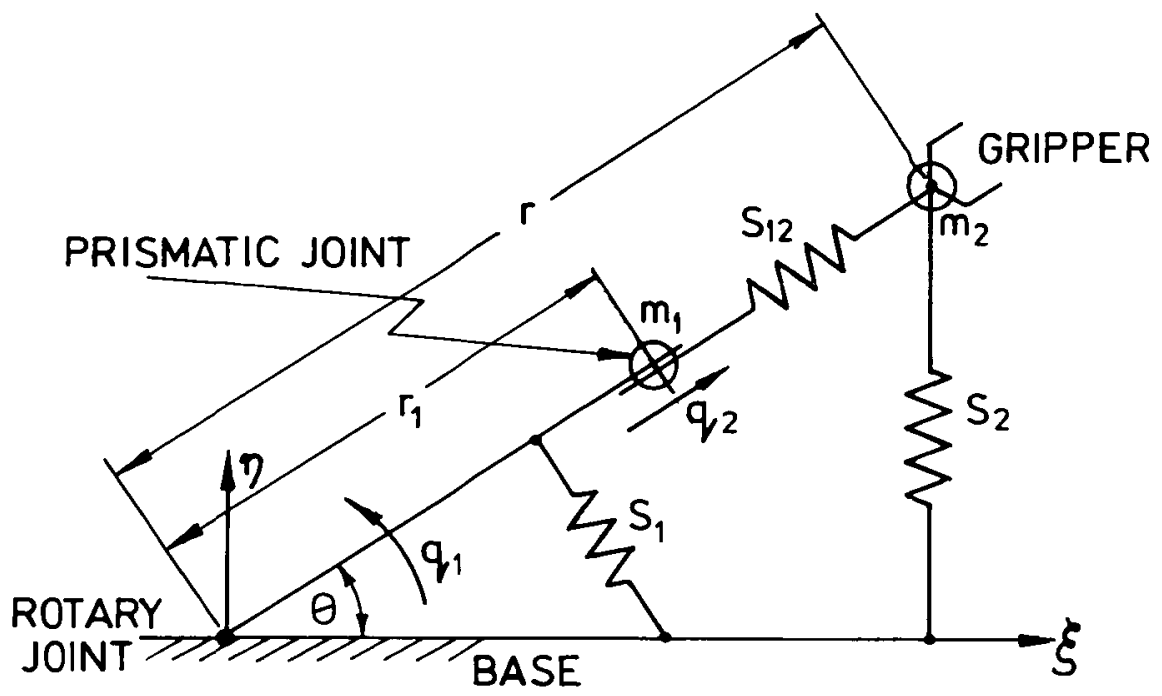

Figure 1

We let the gravity be accompanied by the compensating elastic forces in links or effecting links. To offset the gravity of $9.81 m_{1}$ and $9.81\left(m_{1}+m_{2}\right)$ we set up the springs $S_{1}=a_{1} q_{1}+b_{1} q_{1}^{2}+c_{1} q_{1}^{3} ; \quad S_{2}=a_{2} q_{2}+b_{2} q_{2}^{3}$ with "hardening" effect. Moreover the elastic coupling between the two heavy joints (restoring force for link 2) $S_{12}=a_{12}\left(q_{2}-r_{1}\right)$ is considered proportional to how heavy the joint 1 is: $a_{12}=9.81 m_{1}$. Obviously $S_{12}=-S_{21}$. Hence we have the potential energy

$$
\begin{aligned}
\mathscr{V}= & 9.81 m_{1} r_{1} \sin q_{1}+9.81 m_{2} q_{2} \sin q_{1}+\lambda_{3} q_{1}^{2} / 2+b_{1} q_{1}^{3} / 3+c_{1} q_{1}^{4} / 4 \\
& +a_{12}\left(q_{2}-r_{1}\right) q_{1}-1 / 2 \cdot 9.81 m_{1} q_{2}^{2}+9.81 m_{1} r_{1} q_{2} \\
& +\lambda_{4} q_{2}^{2} / 2+b_{2} q_{2}^{4} / 4 .
\end{aligned}
$$

Then we may calculate the total energy $H=T+\mathscr{V}$ and the Lagrangian $L=T$ $-\mathscr{V}$. Assuming the damping in joints to be $\lambda_{1}\left|\dot{q}_{1}\right| \dot{q}_{1}$ in joint 1 , and $\lambda_{2} \dot{q}_{2}^{2}$ in joint 2 , the Lagrange equations give

$$
\left.\begin{array}{l}
\left(m_{1} r_{1}^{2}+m_{2} q_{2}^{2}\right) \ddot{q}_{1}+2 m_{2} q_{2} \dot{q}_{1} \dot{q}_{2}+\lambda_{1}\left|\dot{q}_{1}\right| \dot{q}_{1}+\lambda_{3} q_{1}+b_{1} q_{1}^{2}+c_{1} q_{1}^{3} \\
\quad+a_{12}\left(q_{2}-r_{1}\right)+9.81\left(m_{1} r_{1}+m_{2} q_{2}\right) \cos q_{1}=u_{1}+K_{1}(t), \\
m_{2} \ddot{q}_{2}-m_{2} q_{2} \dot{q}_{1}^{2}+\lambda_{2} \dot{q}_{2}^{2}+\lambda_{4} q_{2}+b_{2} q_{2}^{3}-a_{12}\left(q_{2}-r_{1}\right) \\
\quad+9.81 m_{2} \sin q_{1}=u_{2}+K_{2}(t),
\end{array}\right\}
$$

where the $K_{1}(t), K_{2}(t)$ represent external perturbations. Denote

$$
\begin{aligned}
& \Phi_{1}=\frac{2 m_{2} q_{2} \dot{q}_{1} \dot{q}_{2}+\lambda_{1}\left|\dot{q}_{1}\right| \dot{q}_{1}}{m_{1} r_{1}^{2}+m_{2} q_{2}^{2}}, \\
& \Phi_{2}=\frac{\lambda_{2}}{m_{2}} \dot{q}_{2}^{2}-q_{2} \dot{q}_{1}^{2}
\end{aligned}
$$


and

$$
\begin{aligned}
& \Pi_{1}=\frac{9.81\left(m_{1} r_{1}+m_{2} q_{2}\right) \cos q_{1}+\lambda_{3} q_{1}+b_{1} q_{1}^{2}+c_{1} q_{1}^{3}+a_{12}\left(q_{2}-r_{1}\right)}{m_{1} r_{1}^{2}+m_{2} q_{2}^{2}} \\
& \Pi_{2}=9.81 \sin q_{1}-\frac{a_{12}}{m_{2}}\left(q_{2}-r_{1}\right)+\frac{1}{m_{2}}\left(\lambda_{4} q_{2}+b_{2} q_{2}^{3}\right) .
\end{aligned}
$$

On letting $\sin q_{1}=q_{1}-q_{1}^{3} / 6, \cos q_{1}=1-q_{1}^{2} / 2, a_{12}=9.81 m_{1}$, we have

$$
\begin{aligned}
& \Pi_{1}=\frac{q_{1}\left(\lambda_{3}-\frac{1}{2} q_{1}+b_{1} q_{1}+c_{1} q_{1}^{2}\right)+9.81 q_{2}\left(m_{1}+m_{2}\right)}{m_{1} r_{1}^{2}+m_{2} q_{2}^{2}} \\
& \Pi_{2}=9.81\left(q_{1}-\frac{1}{6} q_{1}^{3}-\frac{m_{1}}{m_{2}} q_{2}+\frac{m_{1}}{m_{2}} r_{1}\right)+\lambda_{4} q_{2}+b_{2} q_{2}^{3},
\end{aligned}
$$

and finally

$$
\begin{aligned}
& F_{1}=\frac{K_{1}(t)}{m_{1} r_{1}^{2}+m_{2} q_{2}^{2}}, \\
& F_{2}=K_{2}(t) / m_{2}, \\
& \tilde{u}_{1}=\frac{u_{1}}{m_{1} r_{1}^{2}+m_{2} q_{2}^{2}}, \\
& \tilde{u}_{2}=u_{2} / m_{2},
\end{aligned}
$$

which make (6.3) become

$$
\begin{array}{r}
\ddot{q}_{1}+\Phi_{i}\left(q_{1}, q_{2}, \dot{q}_{1}, \dot{q}_{2}, \lambda_{\imath}\right)+\Pi_{\imath}\left(q_{1}, q_{2}, \lambda_{i+1}\right)=F_{\imath}\left(q_{2}, t\right)+\tilde{u}_{i}, \\
i=1,2 .
\end{array}
$$

The reference model is taken as

$$
\ddot{q}_{m i}+\Phi_{m i}\left(q_{m}, \dot{q}_{m}, \lambda_{m i}\right)+\Pi_{m i}\left(q_{m}, \lambda_{m(i+1)}\right)=u_{m i}, \quad i=1,2,
$$

where $\Phi_{m i}=\lambda_{m \imath} \dot{q}_{m \imath}, i=1,2, \lambda_{m i}=$ const. $>0$

$$
\begin{aligned}
& \Pi_{m 1}=\frac{q_{m 1}\left(\lambda_{m 3}-\frac{1}{2} q_{m 1}+b_{1} q_{m 1}+c_{1} q_{m 1}^{2}\right)+9.81 q_{m 2}\left(m_{1}+m_{2}\right)}{m_{1} r_{1}^{2}+m_{2} q_{m 2}^{2}} \\
& \Pi_{m 2}=9.81\left(q_{m 1}-\frac{1}{6} q_{m 1}^{3}-\frac{m_{1}}{m_{2}} q_{m 2}+\frac{m_{1}}{m_{2}}+r_{1}\right)+\frac{1}{m_{2}}\left(\lambda_{m 4} q_{m 2}+b_{2} q_{m 2}^{3}\right)
\end{aligned}
$$

We let $V_{m}\left(x_{m}\right)=H_{m}\left(q_{m}, \dot{q}_{m}\right)=T_{m}\left(q_{m}, \dot{q}_{m}\right)+\mathscr{V}_{m}\left(q_{m}\right)$ be the total energy of the model, and require

$$
\dot{H}_{m}\left(q_{m}(t), \dot{q}_{m}(t)\right)=\sum_{i=1}^{2}\left(u_{m i}-\lambda_{m i} \dot{q}_{m i}\right) \dot{q}_{m i} \leqslant-c,
$$


which is attainable with $u_{m i} \dot{q}_{m i}<\lambda_{m \imath} \dot{q}_{m 1}^{2}$ or $u_{m \imath}=\lambda_{m \imath} \dot{q}_{m l}-k_{\imath} / \dot{q}_{m \imath}, k_{\imath}>0$, $i=1,2$. Now we have

$$
\begin{aligned}
& V_{S}=H_{m}(q, \dot{q})+H_{m}\left(q_{m}, \dot{q}_{m}\right)+a \cdot \alpha, \\
& V_{\eta}= \begin{cases}\left|H_{m}(q, \dot{q})-H_{m}\left(q, \dot{q}_{m}\right)\right|+a \cdot \alpha, \text { on } \overline{C M_{\eta}}, \\
0, \text { on } M_{\eta},\end{cases}
\end{aligned}
$$

where $\alpha=\left(\alpha_{1}, \ldots, \alpha_{4}\right), \alpha_{i}=\lambda_{1}-\lambda_{m v}, i=1, \ldots, 4$, and the adaptive laws (5.5) are

$$
\dot{\alpha}_{i}=\frac{1}{\operatorname{sign} \alpha_{i}} \frac{\partial H_{m}\left(q_{m}, \dot{q}_{m}\right)}{\partial x_{i}}, \quad i=1, \ldots, 4,
$$

where $x_{1}=\left(q_{1}, q_{2}, \dot{q}_{1}, \dot{q}_{2}\right)$, respectively, producing

$$
a \cdot \dot{\alpha}=\dot{H}_{m}\left(q_{m}(t), \dot{q}_{m}(t)\right) \leqslant-c .
$$

Then the control program (5.7) becomes

which holds if

$$
\nabla H_{m}(q, \dot{q}) \cdot(\dot{q}, F+\tilde{u}-\Phi-\Pi)=-c,
$$

$$
\frac{\partial H_{m}(q, \dot{q})}{\partial \dot{q}_{i}} \tilde{u}_{i}=-\frac{\partial H_{m}(q, \dot{q})}{\partial \dot{q}_{i}}\left(F_{1}-\Phi_{i}-\Pi_{i}\right)-\frac{\partial H_{m}(q, \dot{q})}{\partial q_{i}}-c_{1} .
$$

With the terms $\frac{\partial H}{\partial q_{\imath}} m=\frac{\partial T}{\partial q_{i}} m=\frac{\partial T}{\partial \dot{q}_{1}}, F_{\imath}, \Phi_{\imath}, \Pi_{t}$ substituted, the explicit form for $\tilde{u}_{i}=P_{i}(x, \lambda)$ is easily obtained. As shown, the above secure the stabilization and model tracking determined in Definition 3.1. Numerical simulation with the data: $m_{1}=70 \mathrm{~kg}, m_{2}=36 \mathrm{~kg}, r_{1}=0.66 \mathrm{~m}, b_{1}=3, c_{1}=10, b_{2}=2, \lambda_{m 1}=5, \lambda_{m 2}=2$, $\lambda_{m 3}=20, \lambda_{m 4}=\frac{70}{36} \cdot 9.81, \alpha_{1}^{0}=60, \alpha_{2}^{0}=40, \alpha_{3}^{0}=5, \alpha_{4}^{0}=2$ confirms the stabilization and convergence, when the joint system of (6.4), (6.5), (6.7) is solved, subject to $u_{m i}, \tilde{u}_{i}$ determined as specified above.

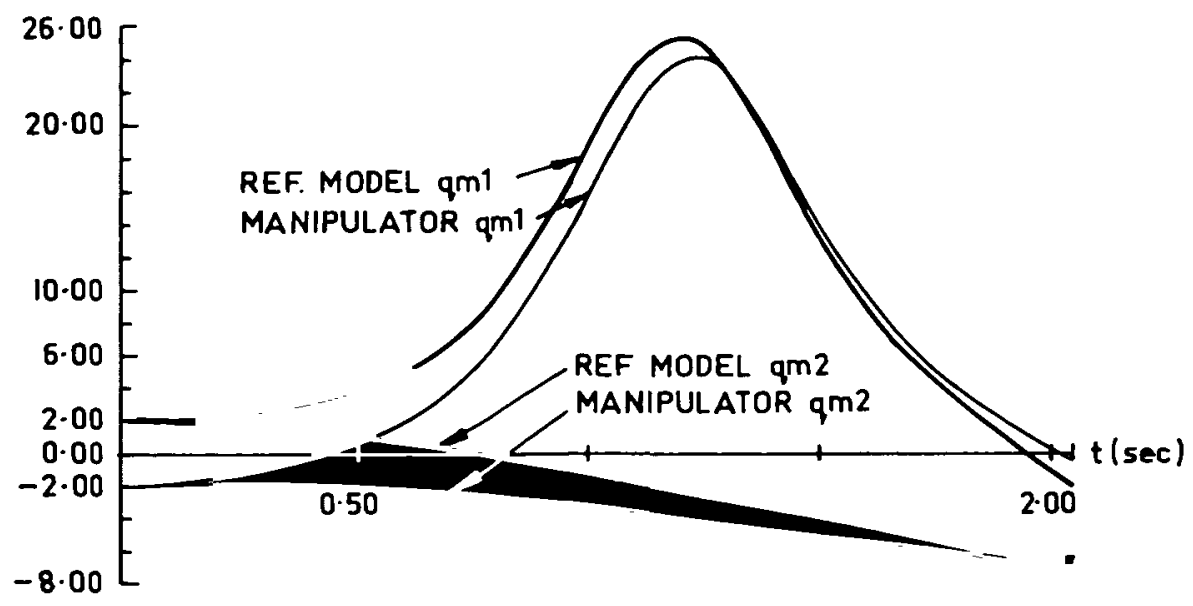

Figure 2 


\section{References}

[1] A. Balestrino, G. De Maria and L. Sciavicco, "An adaptive model following control for robotic manipulators," Trans. ASME Ser. G. J. Dynamic Systems Measurement Control 105 (1985), 143-151.

[2] H. H. Choe and P. N. Nikiforuk, "Inherently stable feedback control of a class of unknown plants," Automatica 7 (1971), 607-625.

[3] S. Dubovsky and D. I. Des Forges, "The application of model referenced adaptive control to robotic manipulators," Trans. ASME Ser. G. J. Dynamic Systems Measurement Control 101 (1979), 193-200.

[4] H. Erzberger, "Analysis and design of model following control," Proc. 1968 JACC, Ann Arbor, Mich., 572-581.

[5] S. B. Gershwin and D. H. Jacobson, "A controllability theory for nonlinear systems," IEEE Trans. A utomat. Control 16 (1971), 37-50.

[6] I. D. Landau, Adaptwe control: the model reference approach (M. Dekker, N. Y., 1979).

[7] G. Leitmann and J. Skowronski, "Avoidance Control," J. Optim. Theory Appl. 23 (1977), 581-591.

[8] G. Leitmann and J. Skowronski, "Note on avoidance control," Optımal Control Appl. Methods 4 (1983), 335-342

[9] K. S. Narendra and S. Tripathi, "Identification and optimization in aircraft dynamics," $J$. Aircraft 10 (1973), 193-199.

[10] J. M. Skowronski, "Liapunov type playability for adaptive physical systems," Proc. Nat. Systems Conf. 1977, PSG Coll. of Techn. India, Q11, 1-5.

[11] J. M. Skowronski, "Parameter and state identification in nonlinearizable uncertain systems," Internat. J. Non-linear Mech. 19 (1984), 345-353.

[12] J. M. Skowronski, "Adaptive identification of models stabilizing under uncertainty," Lecture Notes in Biomathematics 40 (1981), 64-78.

[13] J. M. Skowronski, "Sufficient criterion for synthesable stability of general physical lumped systems," Bull. Acad. Polon. Scl. Ser. Sci. Tech. 14 (1966), 425-428. 